\title{
Relevance of emotioncy in dubbing preference: A quantitative inquiry
}

The International Journal for Translation \& In erpreting Research trans-int.org

\section{Masood Khoshsaligheh}

Ferdowsi University of Mashhad, Iran

khoshsaligheh@um.ac.ir

\section{Reza Pishghadam}

Ferdowsi University of Mashhad, Iran pishghadam@um.ac.ir

\section{Simindokht Rahmani}

Shahid Beheshti University, Iran

simindokht.rahmani@gmail.com

\section{Saeed Ameri}

Ferdowsi University of Mashhad, Iran s.ameri@mail.um.ac.ir

DOI:10.12807/ti.110201.2018.a05

\begin{abstract}
The purpose of this research was to examine the relevance of emotioncy in Persian dubbing preference. Emotioncy deals with senseinduced emotions which can change individuals' interpretation. Approximately two hundred Iranian native speakers of Persian were asked to participate in the study and complete two instruments after viewing five pairs of dubbed segments into Persian. After the originally-designed instruments, emotioncy and dubbing lexical preference scales, had been confirmed for their construct validity using Rasch analyses, structural equation modeling (SEM) was employed to examine the relationship between emotioncy and dubbing lexical preference on the part of the participants who viewed dubbed segments. The analyses revealed that the viewers in this study found a dubbed foreign audiovisual text which contains higher emotioncy lexical items more preferable. This quantitative study recommends supplementary qualitative studies as well as the replication of this design in other language combinations and other modalities of audiovisual translation to complement the findings of this research.
\end{abstract}

Keywords: emotioncy, dubbing, reception, lexicon, audiovisual translation

\section{Introduction}

The commercial success of a feature film as the most prominent type of audiovisual product is reliant on the "ability of that film to sweep the audience into the fictional world" (Wilken \& Kruger, 2016, p. 256). However, maintaining that success, if any, is challenged when the film goes beyond the 
borders of the original speech community and is substantially dependent on its translation to facilitate the international audiences to enjoy the film the same as the original audience did. Media enjoyment, according to Wissmath, Weibel, and Groner (2009), is determined "not only by media characteristics, but also by the media user's personality traits and by the domain-specific interest" (p. 118).

There has been a growing body of research addressing the notion of reception in the discipline of audiovisual translation (AVT). These studies have provided a plethora of useful information for both research and practice. Considering the recent findings, "certain assumptions behind current industry norms and strategies need further empirical testing and demand a more systematic study" (Gambier \& Ramos Pinto, 2016, p. 187); thus, collecting sufficient information in terms of the viewers' perception and assessment of the strategies utilized in AVT becomes of significance (Gambier \& Ramos Pinto, 2016). As asserted by Gambier (2013, p. 51), "what is also needed [in AVT] is more experimental studies on the viewer's processing habits, reading strategies and reception patterns", to offer more real-life applications, such as for practising translators or AVT companies.

Psychology-based studies in the realm of AVT-with a strong focus on subtitling and audio-description - have been conducted since the 1980s. In other words, such investigations have targeted the audience of the products, leading to reception studies (Kruger, 2016). The findings of these studies have offered empirical evidence on how the audiences "respond to the complex semiotic ensemble that they process" during an AVT viewing session (Perego, 2016, p. 157). That being said, the role the subtitles play in comprehension, language acquisition, and language processing has been well documented in the literature thus far (Kruger, 2016, p. 277). Recent research has focused on the effectiveness of subtitling (Perego, Del Missier, Porta, \& Mosconi, 2010; Perego, Missier, \& Bottiroli, 2015), poorly segmented and synchronized subtitles (Lång, Mäkisalo, Gowases, \& Pietinen, 2013; Perego et al., 2010), engagement, presence or enjoyment (Kruger, Sanfiel, Doherty, \& Ibrahim, 2016; Wissmath et al., 2009), among others. It appears, however, that dubbing - the main AVT modality for fiction content in Iran - has been underresearched from a psychological aspect with regard to issues like enjoyment, immersion or emotions.

Dubbing, as the most frequent mode of AVT in Iran, has a long history of over 70 years as all foreign feature films and television series have been dubbed for official national broadcast in Iran. Dubbing is a multifaceted mode of AVT, the success of which mainly depends on several factors such as the appropriate translation of dialogue in addition to synchronization, among others (Chaume, 2007). Thus, it is our hypothesis that due to the nature of the newly emerging concept of emotioncy which refers to a degree of emotion one possesses towards a certain word or concept and deals with sense-related emotions relativizing cognition and perception (Pishghadam, 2016) as well as the fairly long tradition of AVT reception studies from a psychological aspect, the notion can be employed as a criterion for the preference of words used in dubbing. In brief, emotioncy means that our emotion and cognition may change based on the senses from which we receive inputs. With that in mind, controlling numerous intervening variables, the current study aims at investigating the relevance of emotioncy in preference of a certain dubbed animation. 
As an emerging concept in (sensory) psychology, the term emotioncy highlights that senses are hierarchically intertwined with emotions which shape cognition. Interested in the emotional aspect of senses, Pishghadam, Tabatabaeyan, and Navari (2013) drew on Greenspan's (1992) Developmental Individual-differences Relationship-based (DIR) model and coined the term emotioncy. In recent years, the concept has been investigated with respect to a host of variables concerning, language learning and assessment, world reception and cognition which are briefly reviewed in the following. The timely shift to reception studies and audienceship in AVT has brought about a testing ground for the long-held and strictly-followed standards and conventions in the industrial practice of audiovisual adaptation and multimedial localization. In this regard, the concept of emotioncy seems to present a perfectly justified point of view among others to re-evaluate many of those standards, beliefs, and conventions.

The current study aims to investigate whether emotioncy is involved in the Iranian audience's preference of one dubbed version over another. In other words, whether the viewers with higher levels of emotioncy for the lexical choices used in a dubbed version would find the dubbed product more preferable than a parallel version which contains words with lower emotioncy.

\section{Theoretical framework}

\subsection{Emotioncy}

Meaningfulness is believed to boost the acquisition, organization, and retrieval of new knowledge. In order to make it meaningful, as Ausuble (1986) proposed, such new knowledge should be related to and built upon prior knowledge which entails not only the kind of information within the content area of immediate interest, but also parallel information which is outside that area. Bruner (1960, 1986) and Piaget (1954), likewise, brought forth experience and experiential data, and assimilation and accommodation as ways to make new knowledge meaningful. Along with these models of learning, Greenspan (1992) proposed the role of affective factors in enhancing the intellectual faculties. A child's capacity to form meaningful symbols, according to Greenspan and Shanker's (2004) functional/emotional approach to language development, is developed through a series of emotional transformations. Following Greenspan's (1992) developmental, individualdifference, relationship-based (DIR) model of language acquisition, a more recent approach, known as emotion-based language instruction (EBLI), has taken the idea further by underscoring the saliency of emotional capital in second language learning and teaching (Pishghadam, Adamson, \& Shayesteh, 2013; Pishghadam, Tabatabaeyan \& Navari, 2013).

EBLI is a new approach to second language acquisition which underscores creating effective emotional relationships with students and emotionalizing language. Emotionalization, as defined by Pishghadam, Adamson et al. (2013), is building emotions towards lexical items and concepts in a language. This degree of emotions, built and raised through emotionalization, is what EBLI holds as the newly introduced term of emotioncy within its core. In other words, emotioncy is varying levels of emotions evoked in individuals through their senses and towards different items of a language (Pishghadam, Adamson, et al., 2013). The idea that these levels of emotions are actually generated by sensory inputs of different kinds 
(auditory, visual, etc.) is where a distinction is drawn between emotioncy and familiarity. In other words, while familiarity underlines the cognitive aspects of information processing (Piaget, 1954), emotioncy deals with the affective side of it and focuses on emotions stimulated by the type of sensory input one receives. Therefore, according to Pishghadam (2016, May), emotioncy stands on a hierarchical matrix of six levels including Null (0), Auditory (1), Visual (2), Kinesthetic (3), Inner (4), and Arch (5). For instance, if one is a stranger to "asparagus", their emotioncy equals 0 (Null); however, if one has some sort of experience of the word such as having heard of it, seen it, touched/eaten it, or researched on it before, their levels of emotioncy amount to 1 (Auditory), 2 (Visual), 3 (Kinesthetic), 4 (Inner), and 5 (Arch) respectively (Table 1 further clarifies the kinds of emotioncy labeled by Pishghadam, Jajarmi, and Shayesteh (2016). It is important to note that the model is also additive with each level adding up to previous one(s). That is, for example, level two entails level one within itself and is audiovisual and bisensory whereas level one is unisensory. That is why moving up the latter of emotioncy can provide an individual with a more thorough emotional experience of the object or concept.

Table 1: Emotioncy levels and experiences (Adapted from Pishghadam, Jajarmi \& Shayesteh, 2016)

\begin{tabular}{ll}
\hline Levels & Experience \\
\hline Null emotioncy & $\begin{array}{l}\text { When an individual has not heard about, seen, or } \\
\text { experienced an object or a concept. }\end{array}$ \\
\hline Auditory emotioncy & $\begin{array}{l}\text { When an individual has merely heard about an object or a } \\
\text { concept. }\end{array}$ \\
\hline Visual emotioncy & $\begin{array}{l}\text { When an individual has both heard about and seen an } \\
\text { object or action. }\end{array}$ \\
\hline Kinesthetic emotioncy & $\begin{array}{l}\text { When an individual has heard of, seen, and touched the real } \\
\text { object or is in close contact with the person who has } \\
\text { experienced the object or concept. }\end{array}$ \\
\hline Inner emotioncy & $\begin{array}{l}\text { When an individual has experienced an object or a concept } \\
\text { first hand. }\end{array}$ \\
\hline Arch emotioncy & $\begin{array}{l}\text { When an individual has undertaken in-depth research to } \\
\text { obtain additional information about an object or a concept. }\end{array}$ \\
\hline
\end{tabular}

On the hierarchical matrix of emotioncy depicted in Figure 1, Null emotioncy equals the term Avolvement. The lower level emotioncies of Auditory, Visual and Kinesthetic emotioncy that involve learners externally result in Exvolvement with lower levels of internalization of concepts. On the higher end of the spectrum are Arch and Inner emotioncies, which engage learners from the inside, accounting for Involvement which means a deeper internalization of ideas and hence a better understanding of reality. In other words, emotions built by sensory experiences can and do relativize cognition what Pishghadam, Jajarmi, et al. (2016) set forth as sensory relativism. In other words, people with lower levels of emotioncies are exvolved (a coinage to contrast 'involved' as the two extremes of a cline which highlights being engaged in something from outside) from the reality out there, experiencing distal emotions - more exaggerated emotions which may be far from realityabout it and, therefore, a more exaggerated image. As people move up the ladder of the matrix of emotioncy, they get further involved and hold proximal emotions that are closer to reality. It is by means of these very emotions 
created through senses that individuals shape and reshape their idiosyncratic meanings and realities of the world.

Figure 1: Emotioncy levels (Adopted from Pishghadam, 2015)

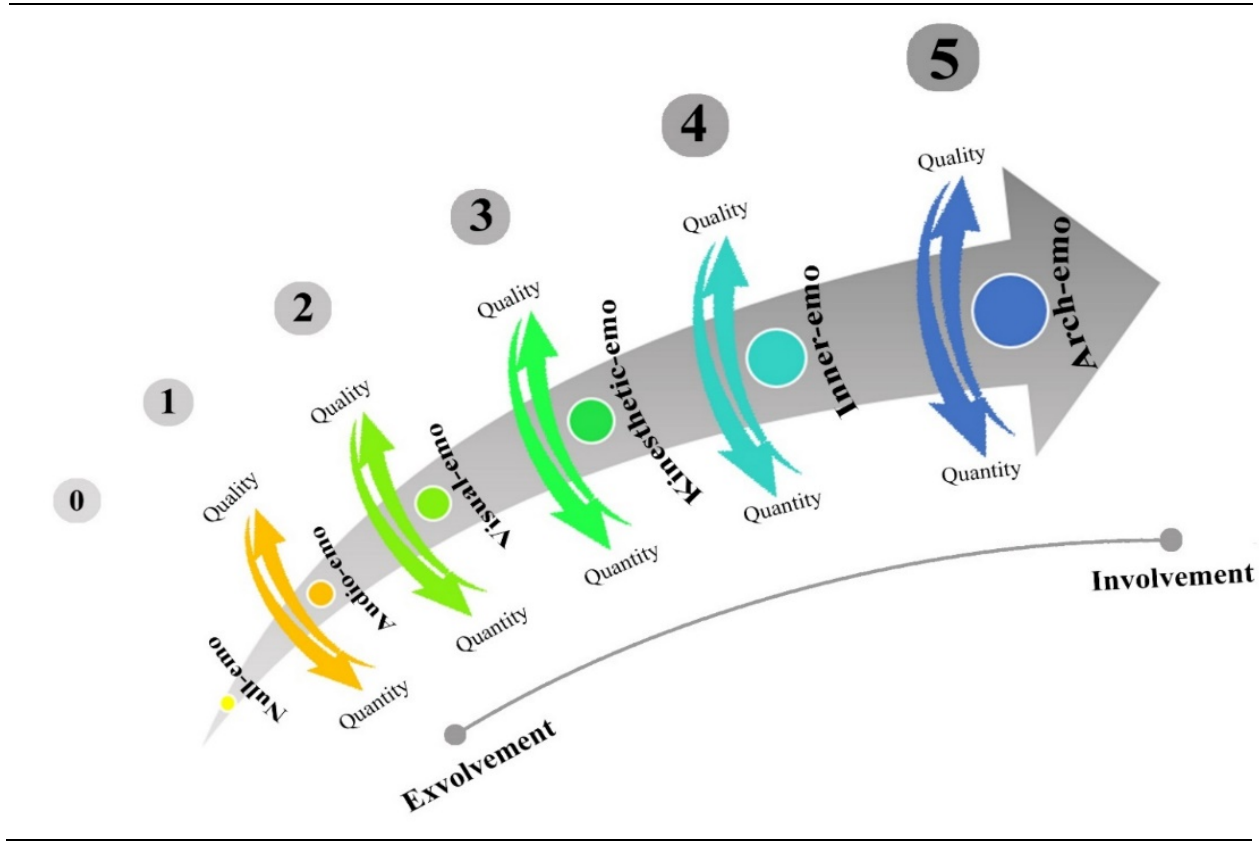

Emotioncy is a rather embryonic concept being delved into and taken further through research. In an empirical study, Pishghadam and Shayesteh (2016) explored the application of emotioncy to vocabulary teaching. The findings revealed higher levels of emotioncy lead to better vocabulary learning and retention. With regard to teaching culture, Pishghadam, Rahmani, and Shayesteh (2017) propounded a hierarchical framework based on the model of emotioncy compartmentalizing the abundant culture teaching techniques already introduced in the literature and implemented in language classrooms. The framework recommends emotionalizing cultural issues and helping learners to move towards higher emotioncy levels in order to give them a proximal emotion which is closer to reality and less inflated. In a similar vein, Pishghadam, Shayesteh, and Rahmani (2016) accentuated emotionalization as beneficial to the learning and teaching environment. To move beyond contextualization, the role of emotionalization was explored in teacher success and teachers who considered students' cognitive and affective experiences were found to be more successful since they tried to involve students through helping them move from "Language and Exvolvement" into "Life and Involvement" (Pishghadam, Shayesteh, et al. 2016, p. 119). Also, Pishghadam, Baghaei, and Seyednozadi (2016), drawing upon the association between senses and emotions, characterized emotioncy as a potential source of test bias and showed that emotional potency of language items bear on their ease of recall by individuals. Finally, in an attempt to further develop the concept of emotioncy, Pishghadam (2016) proposed a new metric to test it. The metric consists of two parts of Sense and Emotion, with Sense referring to the amount of exposure to a concept and Emotion referring to the valence of emotion. In the same study, learners' willingness to communicate (WTC) 
about certain subjects was found to be positively related to their emotioncy scores on those subjects, which attaches the concept of emotioncy to motivation.

To date, studies conducted on emotioncy have mostly stayed within the boundaries of learning and teaching situations. In the present study, however, we intend to move outside these demarcations, focusing on the significance of emotioncy in relativizing cognition and the importance of psychology in AVT reception. Therefore, taking into account that people may experience different emotions based on their culture together with the fact that translation acceptability is culture-specific, emotioncy, with the model it introduces to measure the degree of emotions induced through senses, can be explored as a possible dubbing quality standard in general and dubbing preference specifically.

\subsection{Media and reception}

The emotional reception of audiovisual programs is now being addressed in the realm of AVT and this has provided fruitful findings for the practice and teaching of translation (Rojo, 2017). According to Krcmar and Renfro (2005), enjoyment could be understood in terms of appreciation, attraction, preference and liking, and liking appears to be the most relevant concept to enjoyment which can be defined as "a positively valenced attitude" (p. 5). This, according to Nabi and Krcmar (2004) is "the positive evaluation of a program or character" (p. 290); they also believe that such enjoyment or entertainment, in terms of valence, could influence the message of the media or its experience as a whole. Enjoyment could be specifically examined, for example, in terms of the enjoyment of an episode or genre or even specific elements within a program (Nabi \& Krcmar, 2004).

When it comes to translation, enjoyment has been studied alongside other related concepts such as presence, transportation, and flow. Wissmath et al. (2009), for instance, compared dubbing and subtitling and found no significant difference between the two modalities with regards to the mentioned concepts as possible intervening variables. Furthermore, Kruger et al. (2016) also found that the addition of subtitles in an episode of an American drama series did affect the psychological immersion and enjoyment. That is, watching the film with subtitles increased the level of transportation but it did not significantly affect the enjoyment and presence. Presence has also been examined in audiodescription by Fryer and Freeman (2012) and Fryer, Pring, and Freeman (2013) and recently by Walczak and Fryer (2017a, 2017b). The findings of the experimental studies on audio-description reveal that the description of filmic terms is valued by the blind audiences and can increase the presence while lowering the sound effects did not have any significant effect. But the audiodescription voiced by a human did cause higher levels of presence (Walczak $\&$ Fryer, 2017b).

Emotions have also been the focus of a number of studies on audiodescription. To give an example, Ramos and Rojo (2014), Ramos (2015) and Ramos Caro (2016) studied the emotional experiences of watching audiodescribed films. The investigations aimed to examine the emotional response of the blind audience through heart rates and subjective feelings because "watching movies usually provokes an emotional response in the audience" (Ramos \& Rojo, 2014, p. 133). These studies have emphasized the emotional language in audio-description for elicitation of an emotional response from the blind audience. 
The existing academic literature on dubbing reception shows that researchers have been interested in studying the effects of translation strategies of, among others, humor and culture specific references (Antonini, 2007; Fuentes Luque, 2003), lip-synchronization (Di Giovanni \& Romero Fresco, 2016), comparing dubbing to subtitling (Perego et al., 2015) or attitudes towards expectations (Ameri, Khoshsaligheh, \& Khazaee Farid, $2015,2017)$. Therefore, approaching dubbing from an emotional reception approach is of necessity and in line with subtitling and accessibility reception studies. The study reported on here looks at how dubbing users would prefer the lexical content to be rendered in dubbing and how this is related to the concept of emotioncy.

\subsection{Domestication vs. foreignization}

Translation, for Venuti (1995), tends either to domesticate or foreignize the original text. In the latter case, the translator attempts to convey the foreignness or otherness of the original while in the former this is mitigated or erased in the target text and a fluent translation, by the target culture standards, is produced (Venuti, 1995). Whether the translation should transfer the so-called resistant elements is not a simple question to be directly answered even though dubbing is commonly supposed to reflect natural language; "dubbing primarily consists of domesticating a foreign product to make it seem realistic, credible and in tune with the audience's expectations and experiences" (Chaume, 2012, p. 73).

This question is qualified to be examined by reception studies so as to provide empirical evidence on the preference of the strategies and how well, if at all, they are received (Chaume, 2012, p. 146). Kruger (2013) also argues that the argument or preference of translation scholars towards domestication or foreignization of children's literature has been based on "intuition and personal experience, with no substantial empirical basis" (p. 180). The result of Kruger's (2013) experimental study suggested that some foreignized rendition of lexical items in comparison to domesticated equivalents increased the processing efforts and the children had difficulty in understanding the foreignized elements. In 2007, Liang examined two translation strategies in children's fantasy fiction in a small group of Taiwanese children. The results revealed that children did not find the foreignizing strategy satisfactory (Liang, 2007).

Generally, the previous research on dubbing reveals that Iranian dubbing audiences are interested in a foreignized translation. However, in a recent study on dubbing reception in the Iranian context, the reported mean score was very close to the option no opinion even though it fell within the range of disagreement with a domestication strategy (Ameri et al., 2017). This attitude was also limited to three items, namely, substituting the original songs by local Iranian songs, dubbing the third language spoken in the original program and using pure Persian words. The final two items received a mean range of no opinion and the former received a disagreement mean and made the whole factor containing the three items negative. i.e., disagreement with a domestication strategy. In addition, another qualitative investigation suggested that over-domestication is not preferred by some Iranian viewers of dubbing (Ameri, et al., 2015). The focus of the present study was particularly on a specific part of dubbing, namely the lexicon used in the language of dubbing. 


\section{Method}

\subsection{Participants}

The sample comprised 195 Iranian native speakers of Persian (110 female and 85 male) ranging in age from 13 to $49(M=18.6)$ and studying English as a foreign language in a private language school in Mashhad, a city in northeast Iran. The participants came from different educational background levels with the great majority being high school students and diploma holders $(63.9 \%)$, $11.8 \%$ at bachelor level, and 14\% at MA and Ph.D. levels.

\subsection{Instruments}

\subsubsection{Emotioncy Scale}

The self-report scale of emotioncy was employed to investigate and measure the participants' emotioncy, ranging from a score of 0 to 50 (see Figure 2), towards each of the two Persian equivalent forms for the English words examined in this study. The scale is in Persian (participants' L1) and consists of 6 points: I have never come across this word (0); I have heard someone use it (1); I have heard and seen it in print (2); I have heard, seen and been in contact with someone using it (3); I have heard, seen, and been in contact with someone using it and used it myself (4); and I have heard, seen, been in contact with someone using it, used it myself, and done research on it (5). Each point on this scale includes two Likert-type sections of "frequency" and "emotion", each varying from 1 (very little) to 5 (a lot) and 1 (very bad) to 5 (very good) respectively. Then, using the new metric proposed by Pishghadam (2016), the total emotioncy scores were calculated by multiplying the sense score by the summation of emotion and frequency score: Emotioncy=sense (emotion +frequency). For instance, if a given participant has filled out the emotioncy scale for Blueberry as: I have experienced having studied and getting a low mark nonetheless (sense score=4). I feel good about it (emotion score $=4$ ) and this has happened to me very often (frequency score=5). His total emotioncy score would be 36 .

Figure 2: Model of emotioncy (Adapted from Pishghadam, 2016)

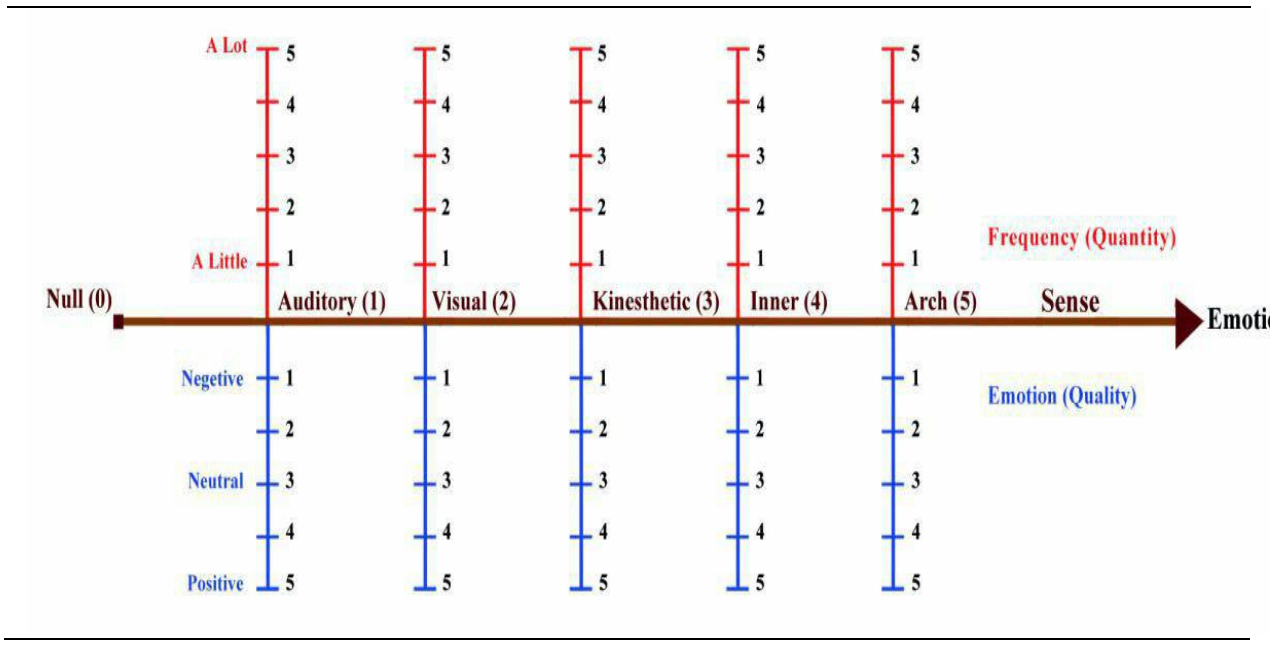

To confirm the uni-dimensionality of the frequency section of the emotioncy, scale Rasch measurement employing WINSTEPS software was 
applied. The results revealed that all the items fitted the model with an item separation index of 5.01 (item reliability=.83), and a person separation index of 5.11 (person reliability $=.94$ ). Moreover, the emotion section of the emotioncy scale was found to be uni-dimensional. The outcomes showed that the item separation index was 5.33 with an item reliability of .91 , and the person separation index was 5.11 with a person reliability of .93 .

\subsubsection{Dubbing lexicon preference scale}

The second scale designed by the authors consisted of seven sections each entailing two Persian equivalents for the original English words, employed in the animation and were utilized in this study, which made a total number of 14 items. For each item, there is a 9-point scale ranging from 1 (very poor) to 9 (excellent). To substantiate the validity of this scale, another Rasch was run. The overall analysis of the items showed that except for the two items related to the word conductor, all items fitted the Rasch model. Consequently, the validation process left the scale with 12 items (6 sections). Overall, the analysis yielded an item separation index of 6.01 with an item reliability of .93 , and a person separation index of 4.25 with a person reliability of .84 .

\subsection{Procedures}

To gather the required ingredients for the questionnaires, the American animated feature named Zootopia (2016) together with five professional Persian dubbed versions were carefully watched several times with the aim of spotting ten vocabulary items within ten short segments. The criterion for choosing the words was the existence of two or more Persian equivalents with varying degrees of frequency of use in Persian. For instance, the vocabulary item "ecosystem" was picked since there exist two different words for that in Persian: /ekosistem/ which is very common among Persian speakers, and /nezaam-e zist/ which is not very frequently used or heard in Persian. From the five professional dubbed versions available in Persian, the researchers decided on two dubbed versions for each of the original segments, contextualizing the selected keywords. The choice of the two dubbed versions was made based on the degree to which they shared the exact same features. The aim was for the two dubbed versions to be parallel in every aspect such as their audiovisual quality or the wording and translation of the whole segment, with the exception of the one specific term picked. Following the example above, the dubbed segments chosen for the word "ecosystem" were cut from the two Persian versions containing the two different Persian translations while being precisely the same in every other aspect. The selected Persian lexical items were then put into both the Emotioncy Scale and Dubbing Lexicon Preference Scale. Prior to data collection, the two questionnaires were piloted with 20 potential participants to ensure the distinction between the Persian lexical pairs and disambiguate the scales. Based on the feedback received, seven pairs remained, and the rest were removed from the questionnaires.

The data collection entailed three phases. In the first part, the Emotioncy Scale was distributed to the participants studying English as a foreign language in Mashhad. Next, after a period of approximately two weeks, the same participants were required to mark the preference of each pair of dubbed segments on the Dubbing Preference Scale right after watching the two dubbed versions containing the selected words. Finally, after approximately 10 days, 30 of the participants were asked to mark the preference of the same 
seven pairs of Persian lexical items again, this time without watching the segments.

\section{Results}

\subsection{Descriptive information}

Table 2 illustrates the seven selected words in the original film, their two alternative equivalents used in the two dubbed versions into Persian and their frequency information in terms of preference by the participating audience. Additionally, a brief explanatory note is provided which shortly describes how the two alternatives and to what extent differ from one another although they are readily equivalents for the same foreign word.

What can be inferred from Table 2 is that the participants were more inclined towards the more familiar terms. For example, the word Cafeteria has two equivalents, one has been borrowed from English while the other is derived from Persian language roots. The number of the participants who chose the first alternative outnumbered those who chose the second alternative as it looks less foreign. The expression delusion of grandeur was translated literally in the first dubbing while more creatively localized in the second one. The participants preferred the latter. As far as false advertisement is concerned, the first option was more favored by the audience as this translation is more frequently used than the second one and it feels more natural in Persian.

Table 2: Descriptive information of the original and equivalent words

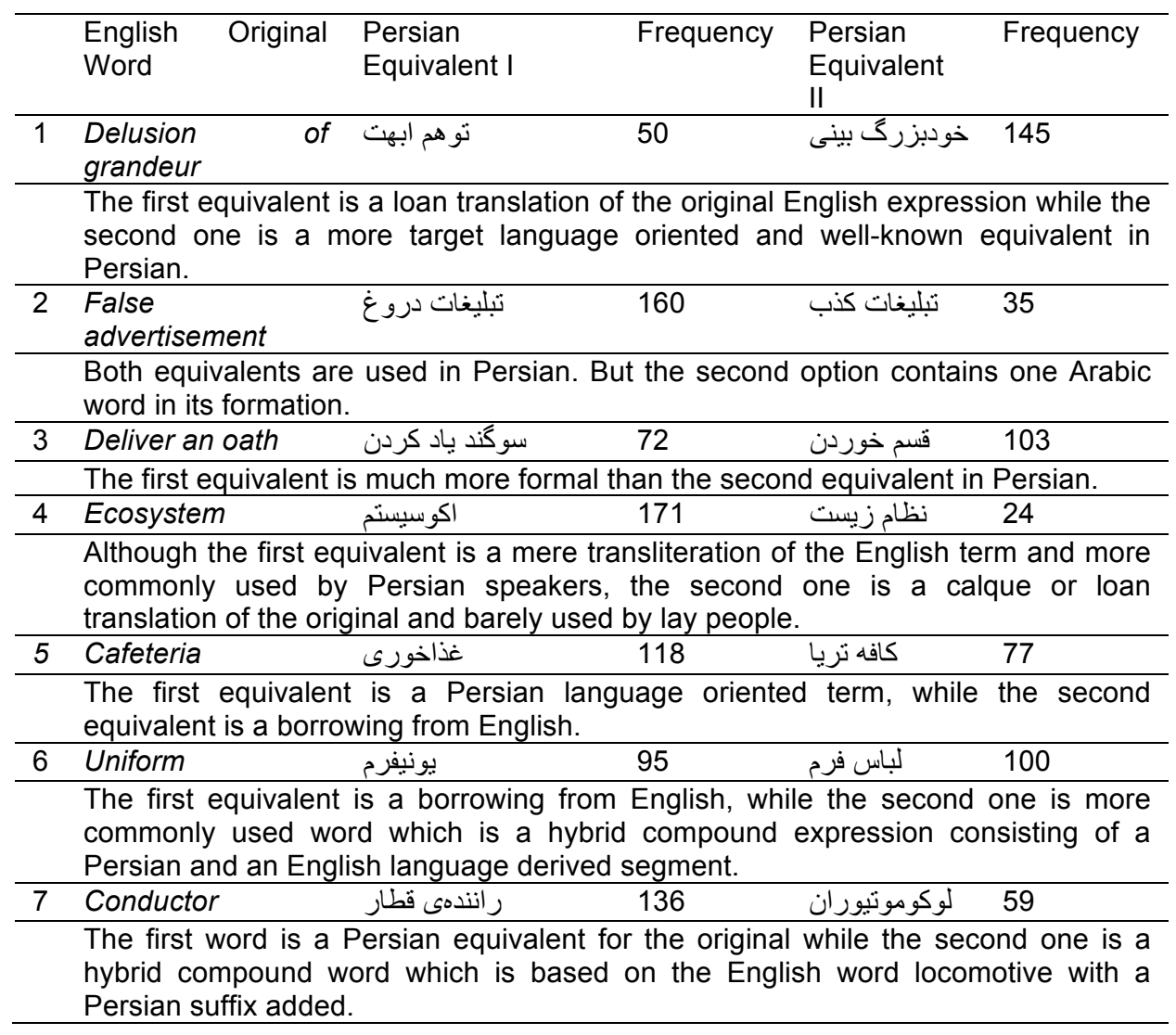


Concerning the third word, the second alternative has been predominantly chosen because this is more commonly used in spoken language and close to the oral registers. Ecosystem is a special case here. The first equivalent for it is a borrowing and the second one is a calque and the first one is more widely used than the second one. Additionally, the second option is not a very natural translation and there exists a better equivalent for it in Persian. The participants equally chose the translation options for Uniform even though the second one was slightly more preferred. The first alternative for this word is a borrowing from English and the second seems to be a Persian language-based equivalent. Last but not least, the participants opted for the first equivalent of the term conductor which is a more localized version than the second that refers to the term locomotive. Overall, the review of the above information reveals that the participants are more interested in and opt for equivalents which they feel are more natural, or with which they are in touch.

The frequencies reported in this part are in line with what is depicted later in Table 3 and Figure 3, highlighting the fact that the words preferred by a higher number of participants are those holding higher emotioncy scores as well.

Table 3 presents the descriptive results along with the related correlation estimates between all the variables.

Table 3: Descriptive statistics for the variables

\begin{tabular}{|c|c|c|c|c|c|c|}
\hline Variables & Mean (SD) & 1 & 2 & 3 & 4 & 5 \\
\hline 1. Avolvement & $.72(.23)$ & 1 & & & & \\
\hline 2. Exvolvement & $6.1(1.2)$ & $.11^{*}$ & 1 & & & \\
\hline 3. Involvement & $3.1(1.9)$ & .03 & $.21^{* *}$ & 1 & & \\
\hline 4. Emotioncy & $141(16.3)$ & .09 & $.23^{* *}$ & $.52^{* *}$ & 1 & \\
\hline 5. Dubbing Lexicon & $29.3(4.9)$ & $-.41^{* *}$ & $12^{*}$ & $48^{* *}$ & $.49^{*}$ & 1 \\
\hline
\end{tabular}

As depicted in Table 3, dubbing lexicon variable is negatively and significantly related to avolvement $(\mathrm{r}=.41, p<.01)$, and positively and significantly related to exvolvement $(\mathrm{r}=.12, \mathrm{p}<.05)$, involvement $(\mathrm{r}=.48, p$ $<.01)$ and emotioncy $(\mathrm{r}=.49, p<.01)$. Moreover, the correlation coefficients illustrate a less significant relation between lexical choice in dubbing and exvolvement compared to the rest.

There are also significant positive correlations between avolvement and exvolvement $(\mathrm{r}=.11, p<.05)$, exvolvement and involvement $(\mathrm{r}=.21, p<$ $.01)$, emotioncy and exvolvement $(\mathrm{r}=.23, p<.01)$, and emotioncy and involvement $(\mathrm{r}=.52, p<.01)$. The patterns demonstrate relations between each type of emotioncy (avolvement, exvolvement, and involvement) and its neighbouring type only; there is no significant correlation between avolvement and involvement.

\subsection{Inferential statistics}

\subsubsection{SEM parameter estimates}

The SEM model below (Figure 2) demonstrates the latent variables of "emotioncy", including the observed variables of frequency and emotion; "avolvement", involving the observed variable of null emotioncy; "exvolvement", entailing the observed variables of auditory, visual and kinesthetic emotioncies; and "involvement", comprising the observed variables of inner and arch emotioncies. There is also the observed variable of Translation \& Interpreting Vol 10 No 1 (2018) 
dubbing lexicon depicted in the model. The numbers next to the arrows show the role each variable plays in determining the variance in the target variable. That is, emotioncy accounts for $17 \%$ of the dubbing lexicon preference variance $\left(\mathrm{R}^{2}=.41\right)$, and involvement and avolvement explain $14 \%\left(\mathrm{R}^{2}=.38\right)$ and $10 \%\left(\mathrm{R}^{2}=-.32\right)$ of the dubbing lexicon preference variance respectively. That is exvolvement does not have a significant contribution to dubbing lexicon preference $\left(\mathrm{R}^{2}=.09\right)$. Consequently, it can be inferred from the model that emotioncy and its two types of involvement and avolvement are strong explanatory factors of dubbing lexicon preference.

Figure 3: Model of the relationship between emotioncy, involvement, exvolvement, avolvement, and lexical choice in translation for dubbing

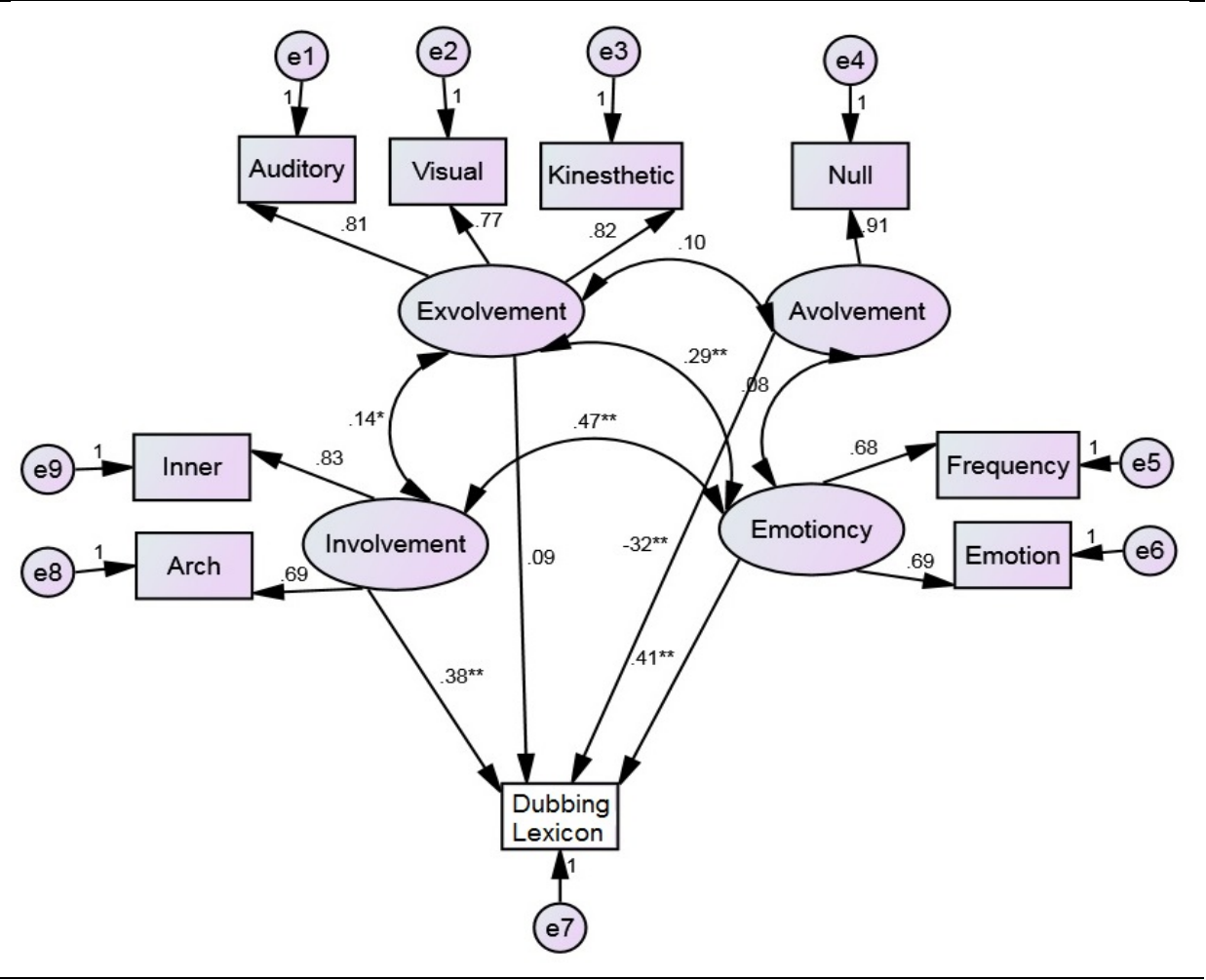

To verify the adequacy of the model, the goodness of fit indices was calculated using Amos. Five indices are presented in Table 4: chi-square index divided by the degrees of freedom $\left(\chi^{2} / d f\right)$ which should be less than 3 (Ullman, 2001), Goodness of Fit Index (CFI) and Tucker-Lewis Index (TLI) which should be over .90, and Root Mean Square Error of Approximation (RMSEA) which should be equal or less than 06 (Schumacker \& Lomax, 2004). Based on the results illustrated in Table 2, the model fits the data adequately.

Table 4: Goodness of fit indices

\begin{tabular}{llllll}
\hline Fit index & $\mathrm{X}^{2} / \mathrm{df}$ & GFI & TLI & CFI & RMSEA \\
\hline Model & 2.45 & .91 & .90 & .93 & .05 \\
\hline
\end{tabular}




\section{Discussion}

This quantitative research mainly aimed to explore the relevance of the recently emerging notion of emotioncy as an indicator of the preference of dubbing overall through the preference of the lexical items used in a dubbed version in particular. The results of the study, conducted in the context of Iran as a Persian speaking and traditionally and predominantly a dubbing country, seem to indicate that emotioncy has a significant positive effect on the preference of for the Iranian audience through the preference of the lexical items used in the dubbed version.

It appears that the participants mainly showed interest towards the more natural and familiar words rather than those that are the result of literal translation or transliteration of the original term or expression. The analyses reveal that preferences are mainly driven by more common words and forms - those that are more naturally occurring in Persian and probably look less foreign. While some scholars have talked about expectations, quality standards, preferences or the acceptability of dubbed products (e.g., BañosPiñero \& Chaume, 2009; Chaume, 2007, 2012), they all can be supported by empirically-grounded studies on audiences' opinions. Consequently, this research seems to be one of only a few empirical studies on dubbing reception from a psychological perspective where lexical choices were evaluated according to the users' preferences and emotioncy.

The findings also indicate that the audience are more inclined to prefer a dubbed product when they have been involved with the lexicon used in the rendition, that is, when they have directly experienced the objects or concepts (Inner emotioncy) or better still have researched them to obtain additional information (Arch emotioncy). In contrast, when the viewers are exvovled with the vocabulary items; that is, they have merely heard about (auditory emotioncy) or have also seen an object or a concept (visual emotioncy) or even have touched the real object (kinesthetic emotioncy), they do not seem to be inclined at all to find the lexical choices of the dubbing and the dubbing itself necessarily preferable.

By the same token, the audience do not tend to find dubbed audiovisual programs preferable when they have been avolved with the lexicon used in the rendition, that is, when they have not heard about, seen, or experienced the object or concept (null emotioncy). This clearly shows how viewers' choices are guided by their cognition. This is perhaps a possible germination for a new generation of research outlook on AVT reception.

The above findings seem to be justified in the light of cognitive psychology and in particular cognitive load theory (Kalyuga, 2009; Plass, Moreno \& Brünken, 2010). It can be reasonably assumed that the audiences are more involved with lexical choices which have more personal relevance to them, and when such vocabulary items are used in AVT such as dubbing, it requires less cognitive load for the information processing of the concepts rendered, and as such they are preferable to other equivalents. It is easily conceivable that people are more mindful of words representing concepts with which they have been in close contact. Naturally they would be more readily processed in the individuals' working memory when heard on a dubbed or original movie or other types of audiovisual text for that matter. Dubbing requires the audience to focus on images and listen to the information simultaneously, because of the multimodal nature of audiovisual texts, as a result of which cognitive overload may occur. To reduce such rather 
extraneous cognitive load, findings suggest, it is helpful to use high-emotioncy words in the dubbing. This would also help with the limited thinking situation the audience is put in while watching the dubbed video. Better put, when individuals are involved in the words used in the dubbed version, they are more able to relate to the text and make sense of it. Moreover, it makes sense to assume that the less cognitive engagement is needed the more the dubbed version provides them with a more enjoyable experience.

In comparison with a movie made in the original culture and language, watching a dubbed foreign movie or television series mainly involves a distant culture with an exotic textual grid. This then naturally takes its toll on the part of the target audience to fully comprehend the events, appreciate the details and nuances of the story and enjoy the fiction as entertainment. Watching a dubbed product resembles a limited thinking situation in which there is not much time to process information and the viewers are more successful in deriving the meaning of the language containing high-emotioncy lexicon from the context with the least cognitive engagement. Thus the audiences seem to tend to find high-emotioncy words in dubbing more preferable in comparison to the lower emotioncy words, as the high-emotioncy words might lighten the cognitive load. The present paper is also a good response to Kruger's (2016) call for the application of well-established methodologies or theories in the field of psycholinguistics to research AVT, so the two fields can reciprocate in contributing to one another.

\section{Conclusion}

Drawing on the findings of this study, it can be reasonably concluded that high-emotioncy words appear to be the more preferable choice for use in translation for dubbing, as they seem to be more preferable on the part of the audience. Watching foreign movies with low emotioncy words could detract the audience's immersion into and enjoyment of the dubbed program.

In line with Chesterman's (2016 [1997]) expectancy norms, an implication of this study can be recommending the consideration of the level of emotioncy of the lexical choices in translation for dubbing in order to meet audience expectations. This also makes sense given translated dialogues in dubbing should be realistic and credible (Chaume, 2012).

This pioneering yet preliminary research serves as a pilot study introducing and exploring the concept of emotioncy in dubbing and clearly requires other supplementary research to make up for its limitations and delimitations. As this paper considered animation as stimuli and included only young participants, children and other viewers should also be examined in future research. To understand the notion of domestication and foreignization or naturalness more deeply, it is suggested these translation strategies poles be investigated in the light of culture specific references. Examining emotioncy alongside variables from media psychology such as presence and transportation could offer interesting evidence in the realm of dubbing. It is recommended that other research investigates the audiences' conception and perception of the notion of emotioncy using in-depth retrospective interviews or think aloud protocols to achieve deeper insights into the relevance of the concept in dubbing. More studies are also recommended to document the effects of emotioncy in other language combinations as well as in other modes of AVT such as subtitling, voice-over, and audio-description. 


\section{References}

Ameri, S., Khoshsaligheh, M., \& Khazaee Farid, A. (2015). هنجار هاى انتظارى بينندكان [Investigating expectancy norms in dubbing in Iran: An exploratory study]. Iranian Journal of Translation Studies, 13(3), 21-37.

Ameri, S., Khoshsaligheh, M., \& Khazaee Farid, A. (2017). The reception of Persian dubbing: A survey on preferences and perception of quality standards in Iran. Perspectives. doi:10.1080/0907676X.2017.1359323

Antonini, R. (2007). SAT, BLT, Spirit Biscuits, and the Third Amendment: What Italians make of cultural references in dubbed texts. In Y. Gambier, M. Shlesinger, \& R. Stolze (Eds.), Doubts and directions in translation studies: Selected contributions from the EST Congress, Lisbon 2004 (pp. 153-167). Amsterdam: Netherlands: John Benjamins.

Ausubel, D. P. (1968). Educational psychology: A cognitive view. New York, NY: Holt, Rinehart and Winston.

Baños-Piñero, R., \& Chaume, F. (2009). Prefabricated orality: A challenge in audiovisual translation. Intralinea, 6, 1-10.

Bruner, J. (1986). Actual minds, possible worlds. Cambridge, MA: Harvard University Press

Bruner, J. S. (1960). The process of education. New York: Vintage Books.

Chaume, F. (2007). Quality standards in dubbing: A proposal. TRADTERM, 13, 7189.

Chaume, F. (2012). Audiovisual translation: Dubbing. Manchester, England: St. Jerome.

Chesterman, A. (2016 [1997]). Memes of translation: The spread of ideas in translation theory (Revised ed.). Amsterdam, Netherlands: John Benjamins.

Di Giovanni, E., \& Romero Fresco, P. (2016, February). Are we all together across languages? An eye tracking study of original and dubbed films. Paper presented at the Conference of Linguistic and Cultural Representation in Audiovisual Translation, Rome, Italy.

Fryer, L., \& Freeman, J. (2012). Presence in those with and without sight: Audio description and its potential for virtual reality applications. The Journal of Cybertherapy and Rehabilitation, 5(1), 15-23.

Fryer, L., Pring, L., \& Freeman, J. (2013). Audio drama and the imagination: The influence of sound effects on presence in people with and without sight. Journal of Media Psychology, 25(2), 65-71. doi:10.1027/1864-1105/a000084

Fuentes Luque, A. (2003). An empirical approach to the reception of AV translated humour: A case study of the Marx Brothers' 'Duck Soup'. The Translator, 9(2), 293-306. doi:10.1080/13556509.2003.10799158

Gambier, Y. (2013). The position of audiovisual translation studies. In C. Millán \& F. Bartrina (Eds.), The Routledge handbook of translation studies (pp. 45-59). Abingdon, England: Routledge.

Gambier, Y., \& Ramos Pinto, S. (2016). Audiovisual translation: Theoretical and methodological challenges- Introduction. Target, 28(2), 185-191. doi: 10.1075/target.28.2.01int

Greenspan, S. \& S. Shanker (2004). The first idea: How symbols, language and intelligence evolved from our primate ancestors to modern humans. Boston: Da Capo, Perseus Books.

Greenspan, S. I. (1992). Infancy and early childhood: The practice of clinical assessment and intervention with emotional and developmental challenges. Madison, CT: International Universities Press.

Kalyuga, S. (2009). Managing cognitive load in adaptive multimedia learning. Hershey, PA.: IGI Global.

Krcmar, M., \& Renfro, S. (2005). Developing a scale to assess media enjoyment. Retrieved from http://citation.allacademic.com $/ / \mathrm{meta} / \mathrm{p}$ _mla_apa_research _citation/0/1/1/8/7/pages11879/p11879-1.php 
Kruger, H. (2013). Child and adult readers' processing of foreignised elements in translated South African picturebooks. Target, 25(2), 180-227. doi:10.1075/target.25.2.03kru

Kruger, J.-L. (2016). Psycholinguistics and audiovisual translation. Target, 28(2), 276-287. doi: 10.1075/target.28.2.08kru

Kruger, J.-L., Sanfiel, M. T. S., Doherty, S., \& Ibrahim, R. (2016). Towards a cognitive audiovisual translatology: Subtitles and embodied cognition. In R. M. Martín (Ed.), Reembedding translation process research (pp. 171-193). Amsterdam, Netherlands: John Benjamins

Lång, J., Mäkisalo, J., Gowases, T., \& Pietinen, S. (2013). Using eye tracking to study the effect of badly synchronized subtitles on the gaze paths of television viewers. New Voices in Translation Studies, 10, 72-86.

Liang, W.-C. (2007). A descriptive study of translating children's fantasy fiction. Perspectives, 15(2), 92-105. doi:10.1080/13670050802153830

Nabi, R. L., \& Krcmar, M. (2004). Conceptualizing media enjoyment as attitude: Implications for mass media effects research. Communication Theory, 14(4), 288-310. doi:10.1111/j.1468-2885.2004.tb00316.x

Perego, E. (2016). History, development, challenges and opportunities of empirical research in audiovisual translation. Across Languages and Cultures, 17(2), 155162. doi:10.1556/084.2016.17.2.1

Perego, E., Del Missier, F., Porta, M., \& Mosconi, M. (2010). The cognitive effectiveness of subtitle processing. Media Psychology, 13(3), 243-272. doi: $10.1080 / 15213269.2010 .502873$

Perego, E., Missier, F. D., \& Bottiroli, S. (2015). Dubbing versus subtitling in young and older adults: Cognitive and evaluative aspects. Perspectives: Studies in Translatology, 23(1), 1-21. doi:10.1080/0907676X.2014.912343

Piaget, J. (1954). The construction of reality in the child. New York, NY: Basic Books.

Pishghadam, R. (2015, October). Emotioncy in language education: From exvolvement $t$ involvement. Paper presented at the 2 nd conference of Interdisciplinary Approaches to Language Teaching, Literature, and Translation Studies. Mashhad, Iran.

Pishghadam, R. (2016, May). Emotioncy, extraversion, and anxiety in willingness to communicate in English. Paper Presented at the 5th International Conference on Language, Education, and Innovation: England, London.

Pishghadam, R., \& Shayesteh, S. (2016). Emotioncy: A post-linguistic approach toward vocabulary learning and retention. Sri Lanka Journal of Social Sciences, 39(1), 27-36.

Pishghadam, R., Adamson, B., \& Shayesteh, S. (2013). Emotion-based language instruction (EBLI) as a new perspective in bilingual education. Multilingual Education, 3(9), 1-16.

Pishghadam, R., Baghaei, P., \& Seyednozadi, Z. (2016). Introducing emotioncy as a potential source of test bias: A mixed Rasch modeling study. International Journal of Testing, 16(5), 1-14.

Pishghadam, R., Jajarmi, H., \& Shayesteh, S. (2016). Conceptualizing sensory relativism in light of emotioncy: A movement beyond linguistic relativism. International Journal of Society, Culture, and Language, 4(2), 11-21.

Pishghadam, R., Rahmani, S., \& Shayesteh, S. (2017). Compartmentalizing culture teaching strategies under an emotioncy-based model. Kasetsart Journal of Social Sciences.

Pishghadam, R., Shayesteh, S., \& Rahmani, S. (2016). Contextualizationemotionalization interface: A case of teacher effectiveness. International and Multidisciplinary Journal of Social Sciences, 5(2), 97-127.

Pishghadam, R., Tabatabaeyan, M. S., \& Navari, S. (2013). A critical and practical analysis of first language acquisition theories: The origin and development. Mashhad, Iran: Ferdowsi University of Mashhad.

Plass, J. L., Moreno, R., \& Brünken, R. (Eds.) (2010). Cognitive load theory. Cambridge, England: Cambridge University Press.

Translation \& Interpreting Vol 10 No 1 (2018) 
Ramos Caro, M. (2016). Testing audio narration: the emotional impact of language in audio description. Perspectives, 24(4), 606-634. doi:10.1080/0907676X.2015. 1120760

Ramos, M. (2015). The emotional experience of films: does Audio Description make a difference? The Translator, 21(1), 68-94. doi:10.1080/13556509.2014.994853

Ramos, M., \& Rojo, A. (2014). "Feeling" audio description: Exploring the impact of AD on emotional response. Translation Spaces, 3, 133-150. doi:10.1075/ts.3.06 ram

Rojo, A. (2017). The role of emotions. In J. W. Schwieter \& A. Ferreira (Eds.), The handbook of translation and cognition (pp. 369-385). Pondicherry, India: John Wiley \& Sons.

Schumacker, R. E., \& Lomax, R. G. (2004). A beginner's guide to structural equation modeling. London: Lawrence Erlbaum Association.

Ullman, J. B. (2001). Structural equation modeling. In B. G. Tabachnick \& L. S. Fidell (Eds.), Using multivariate statistics (4th ed., pp. 653-771). Needham Heights, MA: Allyn \& Bacon.

Venuti, L. (1995). The translator's invisibility. A history of translation. London, England: Routledge.

Walczak, A., \& Fryer, L. (2017a). Creative description: The impact of audio description style on presence in visually impaired audiences. British Journal of Visual Impairment, 35(1), 6-17. doi:10.1177/0264619616661603

Walczak, A., \& Fryer, L. (2017b). Vocal delivery of audio description by genre: measuring users' presence. Perspectives, 1-15. doi:10.1080/0907676X.2017. 1298634

Wilken, N., \& Kruger, J.-L. (2016). Putting the audience in the picture: Mise-en-shot and psychological immersion in audio described film. Across Languages and Cultures, 17(2), 251-270. doi:10.1556/084.2016.17.2.6

Wissmath, B., Weibel, D., \& Groner, R. (2009). Dubbing or subtitling? Effects on spatial presence, transportation, flow, and enjoyment. Journal of Media Psychology, 21(3), 114-125. doi:10.1027/1864-1105.21.3.114 